\title{
Expression of EpCAM in adenoid cystic carcinoma
}

\author{
Seok Joo Lee ${ }^{1}$, Kee Yang $\mathrm{Chung}^{2}$, Ji Eun $\mathrm{Kwon}^{3}$, Sun Och Yoon ${ }^{1}$, \\ SANG KYUM KIM ${ }^{1}$ \\ ${ }^{1}$ Department of Pathology, Yonsei University College of Medicine, Seoul, South Korea; \\ ${ }^{2}$ Department of Dermatology, Yonsei University College of Medicine, Seoul, South Korea; \\ ${ }^{3}$ Department of Pathology, Ajou University School of Medicine, Suwon, South Korea
}

\begin{abstract}
Summary
The mutational landscape of adenoid cystic carcinoma (ACC) is currently being revealed, but further studies are needed to identify biomarkers as therapeutic targets or prognostic factors of ACC. In this study, we investigated the expression of epithelial cell adhesion molecule (EpCAM) in ACCs. We retrospectively collected 83 cases of surgically resected ACCs. Using tissue microarray, we conducted immunohistochemical staining using the antiEpCAM antibody. EpCAM expression was analysed by intensity score and the total immunostaining score. The positivity was $97.6 \%$ (81/83 cases), regardless of the intensity score. A higher histological grade $(p=0.006)$ and specific tumour location (non-salivary gland origin, $p=0.02$ ) showed a correlation with higher EpCAM intensity. Higher EpCAM expression by total immunostaining score was associated with histological grade $(p=0.004)$, distant metastasis $(p=0.004)$ and poorer prognosis (overall survival $p=0.015$ and progressionfree survival $p=0.033$ ). We suggest EpCAM as a candidate prognostic marker and a putative therapeutic target in ACC. Also, ACCs arising from salivary gland and non-salivary gland sites, respectively, might display different pathophysiologies in which EpCAM could play a role.
\end{abstract}

Key words: Adenoid cystic carcinoma; EpCAM; Ber-EP4; biomarker; nonsalivary gland.

Received 21 May, revised 20 August, accepted 23 August 2018

Available online 30 October 2018

\section{INTRODUCTION}

Adenoid cystic carcinoma (ACC) is an uncommon malignancy of the secretory glands and often occurs in the areas of the head and neck, particularly in the salivary glands. ${ }^{1}$ It is characterised by indolent, persistent growth and frequent perineural invasion, local recurrence and a poor long-term prognosis. ${ }^{1,2}$

Immunohistochemical staining is often used to distinguish ACC from other malignancies, especially when histological findings are confusing. ACC expresses both ductal and myoepithelial cell markers, such as CK7, CAM 5.2, calponin, SMA, SMMHC, p63, SOX10, and S100, but their variable expressions in ACC and other malignancies have made them not useful for diagnosis. ${ }^{3,4}$ Currently, c-KIT is widely used as a diagnostic marker, because most ACCs are strongly positive for c-KIT regardless of the histological grade. ${ }^{3,4}$ Many investigators have focused on this receptor as a therapeutic target. ${ }^{5,6}$ However, clinical trials using imatinib, a c-KIT inhibitor, have been unsatisfactory, and several studies have shown that ACCs express wild-type c-KIT. ${ }^{7-10}$ Strong nuclear MYB expression, due to MYB-NFIB translocation, is detected in up to $70 \%$ of ACCs regardless of the site of tumour origin, and MYB appears to be a valuable diagnostic marker for ACC. ${ }^{11-13}$ MYB-NFIB translocation seems to be specific in ACC, and these aberrations may be a critical event in ACC pathogenesis. ${ }^{14}$ However, further studies are needed to uncover the application of MYB-associated genes in therapy.

Epithelial cell adhesion molecule (EpCAM) is a $40 \mathrm{kDa}$ cell surface glycoprotein that is overexpressed in epithelial cancers and, at lower levels, in normal epithelium; therefore, it is considered a tumour marker. ${ }^{15}$ It consists of a large extracellular domain of 242 amino acids (aa), a singlespanning transmembrane domain of 23 aa and a short cytoplasmic domain of 26 aa. The function of EpCAM includes intercellular adhesion, cell proliferation, signalling, migration and differentiation.

EpCAM is overexpressed in certain carcinomas, including colon, pancreas, and breast carcinomas. ${ }^{16-18}$ The high levels of EpCAM in some cancers are related to a poor prognosis, and EpCAM can serve as a prognostic marker. Therefore, the overexpression of EpCAM in tumours makes it an attractive therapeutic target. In the context of skin cancer, EpCAM has been used for diagnostic purposes: the anti-EpCAM antibody Ber-EP4 is a sensitive marker of basal cell carcinoma. ${ }^{19-21}$

Studies regarding EpCAM expression in ACC are relatively scarce. Given the potential diagnostic and therapeutic applications of anti-EpCAM antibodies, we investigated the expression of EpCAM in ACCs. The purpose of this study was to demonstrate the expression of EpCAM in ACC and to verify the clinicopathological features of ACC according to the expression level of EpCAM.

\section{MATERIALS AND METHODS}

Case selection

We included 83 patients with ACC who underwent surgical resection from 1996 to 2013 at Severance Hospital, Korea. Clinical data, including gender, age at diagnosis, tumour location, disease recurrence, metastasis, and survival of patients, were obtained from the patients' medical records. The histological grades of the tumours and perineural invasion were noted from the review of slides and pathology reports. All slides were retrospectively reviewed by two 

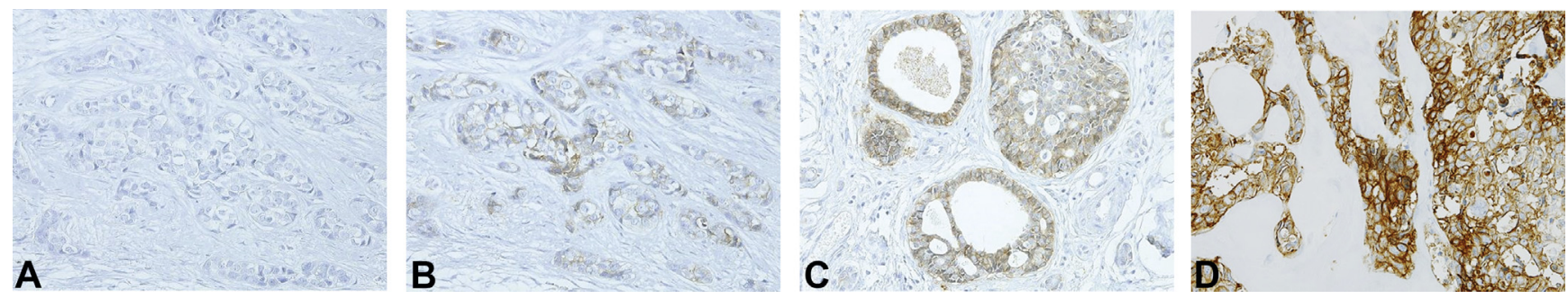

Fig. 1 Examples of the intensity levels of EpCAM in ACC. (A) Negative, 0. (B) Weak, 1+. (C) Moderate, 2+. (D) Strong, 3+.

pathologists (LSJ and KSK), and histological evaluation was conducted on haematoxylin and eosin (H\&E) stained slides. Histological grade was evaluated using the grading system of Perzin/Szanto as follows: 1, predominantly tubular and cribriform component, no solid pattern; 2, predominantly cribriform component or mixed, $<30 \%$ solid component; 3 , marked predominance of the solid component. ${ }^{22,23}$ All methods and experimental protocols using human tissue [formalin fixed, paraffin embedded (FFPE) tissue] were carried out in accordance with relevant guidelines and regulations approved by the Institutional Review Board of Severance Hospital, Yonsei University Health System (4-2015-0872). The informed consent was waived because the IRB decided that this retrospective study showed minimal risk to the patients (risk level I).

\section{Tissue microarray analysis}

A representative area was selected on each $\mathrm{H} \& \mathrm{E}$ slide, and a corresponding spot was marked on the surface of the FFPE block. Using a biopsy needle, the selected area was punched out, and a $3 \mathrm{~mm}$ tissue core was placed into a $6 \times 5$ recipient block. Each tissue core was assigned to a unique tissue microarray location number linked to a database containing other clinical data.

\section{Immunohistochemistry}

FFPE tissue blocks were cut into $4 \mu \mathrm{m}$ sections. Immunohistochemical staining was performed using a Ventana XT automated stainer (Ventana Corporation, USA) with antibodies against EpCAM (clone VU-1D9, 1:1,000; Calbiochem, USA), according to the manufacturer's instructions. Negative control samples were processed without the primary antibody. Positive control tissue was used as per the manufacturer's recommendation.

The expression of EpCAM was semiquantitatively evaluated by intensity score (IS) analysis and by calculating the total immunostaining score (TIS), the product of the intensity score and proportion score (PS). IS represents the estimated staining intensity compared with that of control cells ( 0 , no staining; 1, weak; 2, moderate; 3, strong), and PS describes the estimated area of positively stained tumour cells ( 0 , none; $1,<10 \% ; 2,10-50 \% ; 3,51-80 \% ; 4$, $>80 \%$ ). TIS (IS*PS) ranges from 0 to 12 with only nine possible values $(0,1$, $2,3,4,6,8,9$ and 12). Using TIS, we defined the low-expression group (TIS 0-8) and high-expression group (TIS 9 and 12).

To see the cellular distribution of EpCAM, the expression pattern (membranous or cytoplasmic) was analysed.

\section{Statistical analysis}

All statistical analyses were performed using SPSS software, version 21.0 for Windows (IBM, USA). To analyse each clinicopathological parameter, Student's t-test, Fisher's exact test and Pearson's $\chi^{2}$-test were used, depending on the purpose. Patient survival statistics were analysed using the Kaplan-Meier method and log-rank test, and uni- and multivariate analyses were performed using the Cox regression model. Hazard ratios (HRs) and corresponding 95\% confidence intervals (CIs) were presented. $p<0.05$ was considered to indicate statistical significance.

\section{RESULTS}

\section{Clinicopathological characteristics}

This study included 83 patients with ACC: 53 cases (63.9\%) arose from the salivary gland (46 cases from the major salivary gland and seven cases from the minor salivary gland), and 30 cases $(36.1 \%)$ arose from the non-salivary gland site, including the lacrimal gland, orbit, nasal cavity, pharynx, larynx, auditory canal, breast and trachea. The mean age of

Table 1 Correlation between EpCAM expression by intensity score (IS), total immunostaining score (TIS) analyses and clinicopathological parameters

\begin{tabular}{|c|c|c|c|c|c|c|c|c|c|}
\hline & \multirow[t]{2}{*}{$n$} & \multicolumn{4}{|c|}{ EpCAM expression (IS) } & \multirow[t]{2}{*}{$p$ value } & \multicolumn{2}{|c|}{$\begin{array}{l}\text { EpCAM expression } \\
\text { (TIS) }\end{array}$} & \multirow[t]{2}{*}{$p$ value } \\
\hline & & $\begin{array}{c}\text { Negative }(0) \\
n(\%)\end{array}$ & $\begin{array}{c}\text { Weak (1+) } \\
n(\%)\end{array}$ & $\begin{array}{c}\text { Moderate }(2+) \\
n(\%)\end{array}$ & $\begin{array}{l}\text { Strong }(3+) \\
n(\%)\end{array}$ & & $\begin{array}{c}\text { TIS } \leq 8 \\
n(\%)\end{array}$ & $\begin{array}{c}\text { TIS }>8 \\
n(\%)\end{array}$ & \\
\hline Age (years) & 83 & & & & & 0.464 & & & 0.737 \\
\hline$\leq 50$ & 31 & $1(3.2)$ & $24(77.4)$ & $3(9.7)$ & $3(9.7)$ & & $28(90.3)$ & $3(9.7)$ & \\
\hline$>50$ & 52 & $1(1.9)$ & $33(63.5)$ & $11(21.2)$ & $7(13.5)$ & & $45(86.5)$ & $7(13.5)$ & \\
\hline Gender & & & & & & 0.064 & & & 0.154 \\
\hline Male & 29 & $2(6.9)$ & 19 (65.5) & 7 (24.1) & $1(3.4)$ & & 28 (96.6) & $1(3.4)$ & \\
\hline Female & 54 & $0(0)$ & $38(70.4)$ & $7(13.0)$ & $9(16.7)$ & & $45(83.3)$ & $9(16.7)$ & \\
\hline Location & & & & & & 0.020 & & & 0.484 \\
\hline Salivary gland & 53 & $0(0)$ & $42(79.2)$ & $6(11.3)$ & $5(9.4)$ & & $48(90.6)$ & $5(9.4)$ & \\
\hline Others & 30 & $2(6.7)$ & $15(50.0)$ & $8(26.7)$ & $5(16.7)$ & & $25(83.3)$ & $5(16.7)$ & \\
\hline Histological grade & & & & & & 0.006 & & & 0.004 \\
\hline Grade 1 & 19 & $2(10.5)$ & $14(73.7)$ & $3(15.8)$ & $0(0)$ & & $19(100.0)$ & $0(0.0)$ & \\
\hline Grade 2 & 48 & $0(0)$ & $36(75.0)$ & $8(16.7)$ & $4(8.3)$ & & $44(91.7)$ & $4(8.3)$ & \\
\hline Present & 61 & $1(1.6)$ & $41(67.2)$ & $11(18.0)$ & $8(13.1)$ & & $53(86.9)$ & $8(13.1)$ & \\
\hline Absent & 22 & $1(4.5)$ & $16(72.7)$ & $3(13.6)$ & $2(9.1)$ & & $20(90.9)$ & $2(9.1)$ & \\
\hline Local recurrence & & & & & & 0.234 & & & 0.056 \\
\hline Present & 20 & $0(0)$ & $12(60.0)$ & $3(15.0)$ & $5(25.0)$ & & $15(75.0)$ & $5(25.0)$ & \\
\hline Absent & 63 & $2(3.2)$ & 45 (71.4) & $11(17.5)$ & $5(7.9)$ & & $58(92.1)$ & $5(7.9)$ & \\
\hline Distant metastasis & & & & & & 0.014 & & & 0.004 \\
\hline Present & 30 & $1(3.3)$ & $17(56.7)$ & $4(13.3)$ & $8(26.7)$ & & $22(73.3)$ & $8(26.7)$ & \\
\hline Absent & 53 & $1(1.9)$ & $40(75.5)$ & $10(18.9)$ & $2(3.8)$ & & $51(96.2)$ & $2(3.8)$ & \\
\hline
\end{tabular}



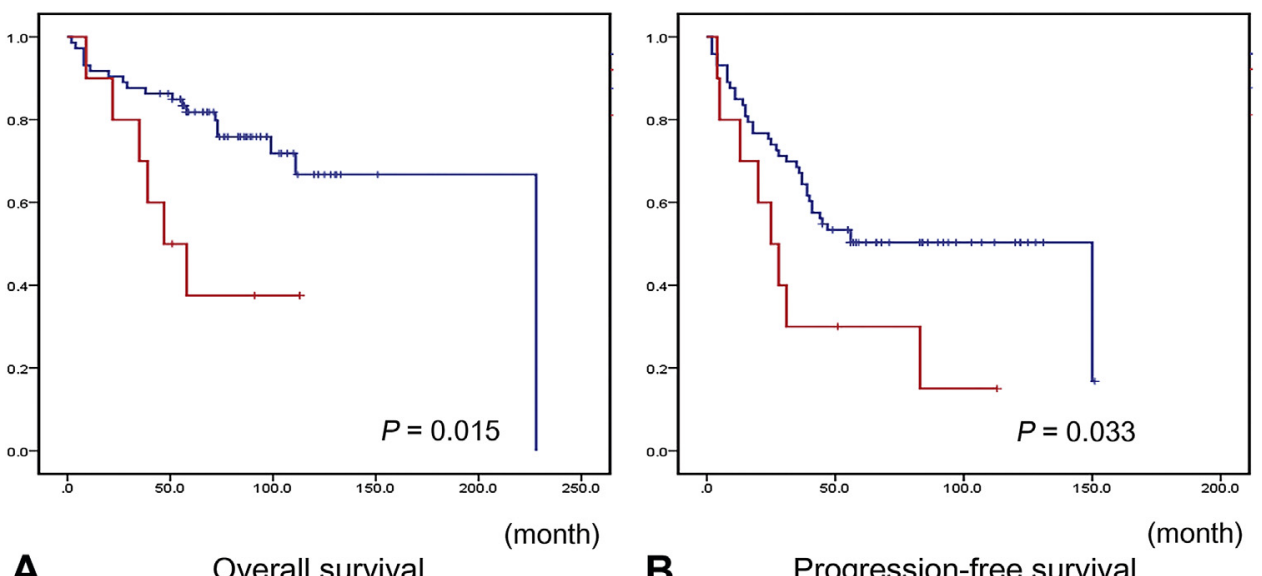

- Low EpCAM

- High EpCAM

A

Overall surviva

B Progression-free survival

Fig. 2 Survival analysis according to the EpCAM expression pattern (TIS $\leq 8$ or TIS $>8$ ) in ACC patients. (A) Overall survival ( $p=0.015$ ). (B) Progression-free survival $(p=0.033)$.

the patients was 53.2 years (age range 20-81 years), and the male-to-female ratio was 1:1.9. According to the histological grade, 19 cases $(22.9 \%)$ demonstrated grade 1, 48 cases $(57.8 \%)$ demonstrated grade 2 , and 16 cases $(19.3 \%)$ demonstrated grade 3 .

\section{EpCAM expression in ACC}

The expression of EpCAM in normal salivary gland tissue was negative or weakly positive in acinar and ductal cells, as previously reported. ${ }^{24}$ Positive staining of EpCAM occurred primarily on the cell membrane and cytoplasm (Fig. 1). The correlation between EpCAM expression by IS, TIS and clinicopathological parameters is listed in Table 1.

The positivity was $97.6 \%$ (81/83 cases), regardless of the intensity score (IS). Negative expression of EpCAM was identified in two cases (2.4\%), weak expression in 57 cases $(68.7 \%)$, moderate expression in 14 cases $(16.9 \%)$, and strong expression in 10 cases $(12.0 \%)$. The number in the low-expression group by TIS was $73(88.0 \%)$ and in the highexpression group was $10(12.0 \%)$. No significant difference was identified between EpCAM intensity and certain clinicopathological parameters such as age at diagnosis, gender, perineural invasion and local recurrence. However, tumour location (non-salivary gland origin, $p=0.02$ ), higher histological grade $(p=0.006)$ and distant metastasis $(p=0.014)$ were related to higher expression of IS. By TIS, a higher EpCAM expression level was also related to the histological grade $(p=0.004)$ and frequent distant metastasis $(p=0.004)$.

\section{Survival analysis}

Kaplan-Meier and Cox regression analyses were performed to discover a link between EpCAM expression in ACC and patient survival. In the Kaplan-Meier analysis, there was a relationship in the patients with higher EpCAM expression (TIS $>8$ ) who had poorer overall survival (OS) and progression-free survival (PFS) than those with a lower TIS level (TIS $\leq 8)$ (OS, $p=0.015$ and PFS, $p=0.033$ ) (Fig. 2). According to Cox regression analysis, the variables with a significant difference in OS rates were male gender $(p=0.009)$, higher histological grade $(p=0.004)$,

Table 2 Univariate and multivariate analyses of clinicopathological variables and EpCAM expression in relation to overall survival and progression-free survival in patients with $\mathrm{ACC}$

\begin{tabular}{|c|c|c|c|c|c|c|}
\hline \multirow[t]{2}{*}{ Risk factors } & \multicolumn{3}{|c|}{ Univariate analysis } & \multicolumn{3}{|c|}{ Multivariate analysis } \\
\hline & HR & $95 \% \mathrm{CI}$ & $p$ value & HR & $95 \% \mathrm{CI}$ & $p$ value \\
\hline \multicolumn{7}{|l|}{ Overall survival } \\
\hline Age $(\leq 50 />50)$ & 1.248 & $0.533-2.920$ & 0.610 & 0.670 & $0.258-1.741$ & 0.411 \\
\hline Gender (male/female) & 0.339 & $0.151-0.765$ & 0.009 & 0.277 & $0.095-0.803$ & 0.018 \\
\hline Location (others/salivary) & 0.894 & $0.410-1.950$ & 0.778 & 1.840 & $0.745-4.546$ & 0.186 \\
\hline Histological grade $(1+2 / 3)$ & 3.170 & $1.431-7.021$ & 0.004 & 2.146 & $0.915-5.031$ & 0.079 \\
\hline Perineural invasion (N/P) & 4.058 & $0.953-17.280$ & 0.058 & 2.164 & $0.448-10.462$ & 0.337 \\
\hline Local recurrence (N/P) & 2.201 & $0.977-4.959$ & 0.057 & 1.525 & $0.555-4.192$ & 0.413 \\
\hline Distant metastasis (N/P) & 3.005 & $1.314-6.873$ & 0.009 & 2.045 & $0.873-4.793$ & 0.100 \\
\hline EpCAM IS $(0-2 / 3)$ & 2.998 & $1.183-7.583$ & 0.021 & 2.908 & $0.896-9.440$ & 0.076 \\
\hline \multicolumn{7}{|l|}{ Progression-free survival } \\
\hline Age $(\leq 50 />50)$ & 0.902 & $0.491-1.658$ & 0.741 & 0.599 & $0.299-1.200$ & 0.148 \\
\hline Gender (male/female) & 0.467 & $0.258-0.847$ & 0.012 & 0.893 & $0.399-2.000$ & 0.784 \\
\hline Location (others/salivary) & 0.876 & $0.481-1.593$ & 0.664 & 1.879 & $0.938-3.766$ & 0.075 \\
\hline Histological grade $(1+2 / 3)$ & 2.131 & $1.113-4.081$ & 0.023 & 0.970 & $0.464-2.030$ & 0.936 \\
\hline Distant metastasis (N/P) & 7.345 & $3.772-14.301$ & $<0.001$ & 7.611 & $3.841-15.083$ & $<0.001$ \\
\hline EpCAM IS $(0-2 / 3)$ & 2.247 & $1.039-4.858$ & 0.040 & 0.956 & $0.344-2.655$ & 0.932 \\
\hline
\end{tabular}


distant metastasis $(p=0.009)$ and strong EpCAM expression $(p=0.021)$ in the univariate analysis (Table 2). EpCAM expression did not affect the OS of ACC patients in the multivariate analysis.

In univariate analysis for PFS, male gender $(p=0.012)$, higher histological grade $(p=0.023)$, perineural invasion $(p=0.012)$, local recurrence $(p<0.001)$, distant metastasis $(p<0.001)$ and strong EpCAM expression $(p=0.04)$ were statistically significant. In the multivariate analysis, EpCAM expression did not affect the PFS. Local recurrence and distant metastasis were identified as independent prognostic factors for PFS. All the statistically significant variables were subjected to the Cox proportional hazards regression model.

\section{DISCUSSION}

Several studies have investigated the expression of EpCAM in various carcinomas. Although EpCAM might be expressed weakly on normal epithelium, it is overexpressed in tumours, including colorectum, oesophagus, liver, prostate, lung, pancreas and breast tumours. ${ }^{18}$ EpCAM overexpression is associated with poorer prognosis in carcinomas of the breast, gallbladder, ovary, ampulla and oesophagus because the molecule functions as an inhibitor of E-cadherin; therefore, it is believed that EpCAM plays a role in metastasis. ${ }^{25-31}$ On the other hand, EpCAM can participate in cell adhesion, and its overexpression is suggested to be linked to better survival in patients with colorectal carcinoma, gastric carcinoma, clear cell renal cell carcinoma and non-small cell lung cancer. ${ }^{32-36}$

We performed immunohistochemical staining of EpCAM on the tissues of 83 ACC samples. Some reports have suggested that the cellular distribution of EpCAM varies by tumour type and histological differentiation of carcinoma; therefore, it might have diagnostic value. ${ }^{37,38}$ However, we identified both membranous and cytoplasmic expression in cancer cells of ACC and it does not have any clinicopathological significance in ACC.

In this study, we found that the overexpression pattern of EpCAM in ACC is associated with a higher histological grade. Furthermore, we could confirm that EpCAM plays a role in tumour distant metastasis in the context of ACC, as previous studies have suggested in other tumours. ${ }^{25-31}$

Phattarataratip et al. studied epithelial cell adhesion molecule expression in various salivary gland neoplasms, including mucoepidermoid carcinoma, adenoid cystic carcinoma, pleomorphic adenoma, and polymorphous low-grade adenocarcinoma. ${ }^{39}$ They included EpCAM and showed different EpCAM expression patterns among salivary gland neoplasms. They demonstrated that decreased EpCAM expression was associated with aggressive features in mucoepidermoid carcinoma, and the ACCs showed negative or weakly positive immunoreactivity to EpCAM, contrary to our result. However, that previous study possessed some limitations in that the number of ACC cases involved in the study was too small and they found that the solid growth area of ACC showed diffuse and strong immunoreactivity to EpCAM.

By immunohistochemistry, the stronger EpCAM staining intensity was more frequently observed in ACCs arising from non-salivary gland sites. We showed that tumour location tends to affect ACC patients' progression-free survival by multivariate analysis. Lin et al. compared the ACCs of salivary and non-salivary origin, and they also found that sinonasal, lacrimal, and tracheobronchial ACCs had significantly worse outcomes than ACCs of the major salivary glands. ${ }^{40}$ The different patients' outcomes between salivary and non-salivary sites of tumour may come from difficulties of diagnosis and complete resection as well as different tumour biologies, but further studies are required to clear these differences according to tumour location.

A previous study investigated the expression of tumourassociated calcium signal transducer 2 (TACSTD2, Trop2), a homolog of EpCAM, in salivary ACC. ${ }^{41}$ Similar to our result of EpCAM in ACC, TACSTD2 overexpression was related to a poor prognosis in patients, although the molecule did not reflect the histological subtype.

The possible prognostic significance of EpCAM overexpression in various cancers has been raised by several investigators. ${ }^{30,42-44}$ Similarly, overexpression of EpCAM was correlated with higher histological grade and distant metastasis and we confirmed strong EpCAM expression is an independent factor affecting ACC patients' survival in the statistical evaluation.

Several clinical trials using various anti-EpCAM antibodies have been investigated despite the controversy. Catumaxomab was approved in Europe to treat malignant ascites in patients with EpCAM-positive carcinomas. ${ }^{45,46}$ Additionally, a few studies using anti-EpCAM antibody showed that it might have an anti-tumour effect. ${ }^{47-49}$ However, the validation of EpCAM as a therapeutic target remains unexplored.

In conclusion, we demonstrated the expression of EpCAM in ACC according to the various clinicopathological conditions. A higher histological grade and specific tumour location (non-salivary gland site) showed higher EpCAM expression, and strong EpCAM positivity was associated with distant metastasis and poorer prognosis. We believe that this study will strengthen the basis for understanding the pathophysiology of ACC and suggests EpCAM as a candidate molecule for a prognostic and therapeutic biomarker.

Conflicts of interest and sources of funding: This research was supported by the Basic Science Research Program through the National Research Foundation of Korea (NRF) funded by the Ministry of Education (grant number: 2016R1D1A1B03931581). The authors state that there are no conflicts of interest to disclose.

Address for correspondence: Sang Kyum Kim, Department of Pathology, Yonsei University Medical Center, 134, Sinchon-dong, Seodaemun-gu, Seoul, South Korea. E-mail: nicekyumi@yuhs.ac

\section{References}

1. Spiro RH, Huvos AG, Strong EW. Adenoid cystic carcinoma of salivary origin. A clinicopathologic study of 242 cases. Am J Surg 1974; 128: 512-20.

2. Nascimento AG, Amaral AL, Prado LA, et al. Adenoid cystic carcinoma of salivary glands. A study of 61 cases with clinicopathologic correlation. Cancer 1986; 57: 312-9.

3. Terada T. Adenoid cystic carcinoma of the oral cavity: immunohistochemical study of four cases. Int J Clin Exp Pathol 2013; 6: 932-8.

4. Zhu S, Schuerch C, Hunt J. Review and updates of immunohistochemistry in selected salivary gland and head and neck tumors. Arch Pathol Lab Med 2015; 139: 55-66.

5. Andreadis D, Epivatianos A, Poulopoulos A, et al. Detection of C-KIT (CD117) molecule in benign and malignant salivary gland tumours Oral Oncol 2006; 42: 57-65.

6. Vila L, Liu H, Al-Quran SZ, et al. Identification of c-kit gene mutations in primary adenoid cystic carcinoma of the salivary gland. Mod Pathol 2009; 22: 1296-302. 
7. Ghosal N, Mais K, Shenjere P, et al. Phase II study of cisplatin and imatinib in advanced salivary adenoid cystic carcinoma. $\mathrm{Br} J$ Oral Maxillofac Surg 2011; 49: 510-5.

8. Hotte SJ, Winquist EW, Lamont E, et al. Imatinib mesylate in patients with adenoid cystic cancers of the salivary glands expressing c-kit: Princess Margaret Hospital phase II consortium study. J Clin Oncol 2005; 23: 585-90.

9. Moskaluk CA, Frierson Jr HF, El-Naggar AK, et al. C-kit gene mutations in adenoid cystic carcinoma are rare. Mod Pathol 2010; 23: 905-6; author reply 906-7.

10. Freier K, Flechtenmacher C, Walch A, et al. Differential KIT expression in histological subtypes of adenoid cystic carcinoma (ACC) of the salivary gland. Oral Oncol 2005; 41: 934-9.

11. West RB, Kong C, Clarke N, et al. MYB expression and translocation in adenoid cystic carcinomas and other salivary gland tumors with clinicopathologic correlation. Am J Surg Pathol 2011; 35: 92-9.

12. Brill 2nd LB, Kanner WA, Fehr A, et al. Analysis of MYB expression and MYB-NFIB gene fusions in adenoid cystic carcinoma and other salivary neoplasms. Mod Pathol 2011; 24: 1169-76.

13. Mitani Y, Li J, Rao PH, et al. Comprehensive analysis of the MYBNFIB gene fusion in salivary adenoid cystic carcinoma: incidence, variability, and clinicopathologic significance. Clin Cancer Res 2010 16: $4722-31$.

14. Ho AS, Kannan K, Roy DM, et al. The mutational landscape of adenoid cystic carcinoma. Nat Genet 2013; 45: 791-8.

15. Schnell U, Cirulli V, Giepmans BN. EpCAM: structure and function in health and disease. Biochim Biophys Acta 2013; 1828: 1989-2001.

16. Baeuerle PA, Gires O. EpCAM (CD326) finding its role in cancer. Br J Cancer 2007; 96: 417-23.

17. Went $\mathrm{P}$, Vasei M, Bubendorf L, et al. Frequent high-level expression of the immunotherapeutic target Ep-CAM in colon, stomach, prostate and lung cancers. Br J Cancer 2006; 94: 128-35.

18. Went PT, Lugli A, Meier S, et al. Frequent EpCam protein expression in human carcinomas. Hum Pathol 2004; 35: 122-8.

19. Tellechea O, Reis JP, Domingues JC, et al. Monoclonal antibody Ber EP4 distinguishes basal-cell carcinoma from squamous-cell carcinoma of the skin. Am J Dermatopathol 1993; 15: 452-5.

20. Jimenez FJ, Burchette Jr JL, Grichnik JM, et al. Ber-EP4 immunoreactivity in normal skin and cutaneous neoplasms. Mod Pathol 1995; 8 $854-8$.

21. Swanson PE, Fitzpatrick MM, Ritter JH, et al. Immunohistologic differential diagnosis of basal cell carcinoma, squamous cell carcinoma, and trichoepithelioma in small cutaneous biopsy specimens. J Cutan Pathol 1998; 25: 153-9.

22. Perzin KH, Gullane P, Clairmont AC. Adenoid cystic carcinomas arising in salivary glands: a correlation of histologic features and clinical course. Cancer 1978; 42: 265-82.

23. Szanto PA, Luna MA, Tortoledo ME, et al. Histologic grading of adenoid cystic carcinoma of the salivary glands. Cancer 1984; 54: $1062-9$.

24. Balzar M, Winter MJ, de Boer CJ, et al. The biology of the 17-1A antigen (Ep-CAM). J Mol Med 1999; 77: 699-712.

25. Spizzo G, Gastl G, Wolf D, et al. Correlation of COX-2 and Ep-CAM overexpression in human invasive breast cancer and its impact on survival. Br J Cancer 2003; 88: 574-8.

26. Gastl G, Spizzo G, Obrist P, et al. Ep-CAM overexpression in breas cancer as a predictor of survival. Lancet 2000; 356: 1981-2.

27. Varga M, Obrist P, Schneeberger S, et al. Overexpression of epithelia cell adhesion molecule antigen in gallbladder carcinoma is an independent marker for poor survival. Clin Cancer Res 2004; 10: 3131-6.

28. Spizzo G, Went P, Dirnhofer S, et al. Overexpression of epithelial cel adhesion molecule (Ep-CAM) is an independent prognostic marker for reduced survival of patients with epithelial ovarian cancer. Gynecol Oncol 2006; 103: 483-8.
29. Fong D, Steurer M, Obrist P, et al. Ep-CAM expression in pancreatic and ampullary carcinomas: frequency and prognostic relevance. $J$ Clin Pathol 2008; 61: 31-5.

30. Stoecklein NH, Siegmund A, Scheunemann P, et al. Ep-CAM expression in squamous cell carcinoma of the esophagus: a potential therapeutic target and prognostic marker. BMC Cancer 2006; 6: 165.

31. Sen S, Carnelio S. Expression of epithelial cell adhesion molecule (EpCAM) in oral squamous cell carcinoma. Histopathology 2016; 68: 897-904.

32. Warneke VS, Behrens HM, Haag J, et al. Members of the EpCAM signalling pathway are expressed in gastric cancer tissue and are correlated with patient prognosis. Br J Cancer 2013; 109: 2217-27.

33. Songun I, Litvinov SV, van de Velde CJ, et al. Loss of Ep-CAM (CO171A) expression predicts survival in patients with gastric cancer. $\mathrm{Br}$ Cancer 2005; 92: 1767-72.

34. Went P, Dirnhofer S, Salvisberg T, et al. Expression of epithelial cell adhesion molecule (EpCam) in renal epithelial tumors. Am J Surg Pathol 2005; 29: 83-8.

35. Goossens-Beumer IJ, Zeestraten EC, Benard A, et al. Clinical prognostic value of combined analysis of Aldh1, Survivin, and EpCAM expression in colorectal cancer. Br J Cancer 2014; 110: 2935-44.

36. Gold KA, Kim ES, Liu DD, et al. Prediction of survival in resected nonsmall cell lung cancer using a protein expression-based risk model: implications for personalized chemoprevention and therapy. Clin Cancer Res 2014: 20: 1946-54.

37. Ogura E, Senzaki H, Yoshizawa K, et al. Immunohistochemical localization of epithelial glycoprotein EGP-2 and carcinoembryonic antigen in normal colonic mucosa and colorectal tumors. Anticancer Res 1998; 18: $3669-75$.

38. Xie X, Wang CY, Cao YX, et al. Expression pattern of epithelial cell adhesion molecule on normal and malignant colon tissues. World $J$ Gastroenterol 2005; 11: 344-7.

39. Phattarataratip E, Masorn M, Jarupoonphol W, et al. Differential expression of epithelial cell adhesion molecule in salivary gland neoplasms. Ann Diagn Pathol 2016; 24: 62-7.

40. Lin YC, Chen KC, Lin CH, et al. Clinicopathological features of salivary and non-salivary adenoid cystic carcinomas. Int J Oral Maxillofac Surg 2012; 41: 354-60.

41. Xia Y, Li B, Gao N, et al. Expression of tumor-associated calcium signa transducer 2 in patients with salivary adenoid cystic carcinoma: correlation with clinicopathological features and prognosis. Oncol Lett 2014; 8: $1670-4$.

42. Kimura H, Kato H, Faried A, et al. Prognostic significance of EpCAM expression in human esophageal cancer. Int J Oncol 2007; 30: 171-9.

43. Osta WA, Chen Y, Mikhitarian K, et al. EpCAM is overexpressed in breast cancer and is a potential target for breast cancer gene therapy. Cancer Res 2004; 64: 5818-24.

44. Kroepil F, Dulian A, Vallbohmer D, et al. High EpCAM expression is linked to proliferation and lauren classification in gastric cancer. $B M C$ Res Notes 2013; 6: 253.

45. Zeidler R, Reisbach G, Wollenberg B, et al. Simultaneous activation of $\mathrm{T}$ cells and accessory cells by a new class of intact bispecific antibody results in efficient tumor cell killing. J Immunol 1999; 163: 1246-52.

46. Ruf $\mathrm{P}$, Gires O, Jager M, et al. Characterisation of the new EpCAM specific antibody HO-3: implications for trifunctional antibody immunotherapy of cancer. Br J Cancer 2007; 97: 315-21.

47. Schmidt M, Scheulen ME, Dittrich C, et al. An open-label, randomized phase II study of adecatumumab, a fully human anti-EpCAM antibody, as monotherapy in patients with metastatic breast cancer. Ann Oncol 2010; 21: 275-82

48. Kurtz JE, Dufour P. Adecatumumab: an anti-EpCAM monoclonal antibody, from the bench to the bedside. Exp Opin Biol Ther 2010; 10: 951-8.

49. Liao MY, Lai JK, Kuo MY, et al. An anti-EpCAM antibody EpAb2-6 for the treatment of colon cancer. Oncotarget 2015; 6: 24947-68. 